Supporting Information

\title{
Nipah virus V protein binding alters MDA5 helicase folding dynamics
}

Nicole D. Wagner ${ }^{1,3^{*}}$, Hejun Liu ${ }^{1,2,4^{*}}$, Henry Rohrs ${ }^{3}$, Gaya K. Amarasinghe ${ }^{2}$, Michael L. Gross $^{3}$, and Daisy W. Leung ${ }^{1,2}$

'Division of Inf ectious Diseases, John T. Milliken Department of Medicine, Washington University School of Medicine, St. Louis, MO 63110, USA

${ }^{2}$ Department of Pathology and Immunology, Washington University School of Medicine, St. Louis, MO 63110, USA

${ }^{3}$ Department of Chemistry, Washington University in St. Louis, St. Louis, MO 63130, USA

${ }^{4}$ Current address: Department of Integrative Structural and Computational Biology, The Scripps Research Institute, La Jolla, CA 92037, USA

*equal contribution

Corresponding author: dwleung@wustl.edu 


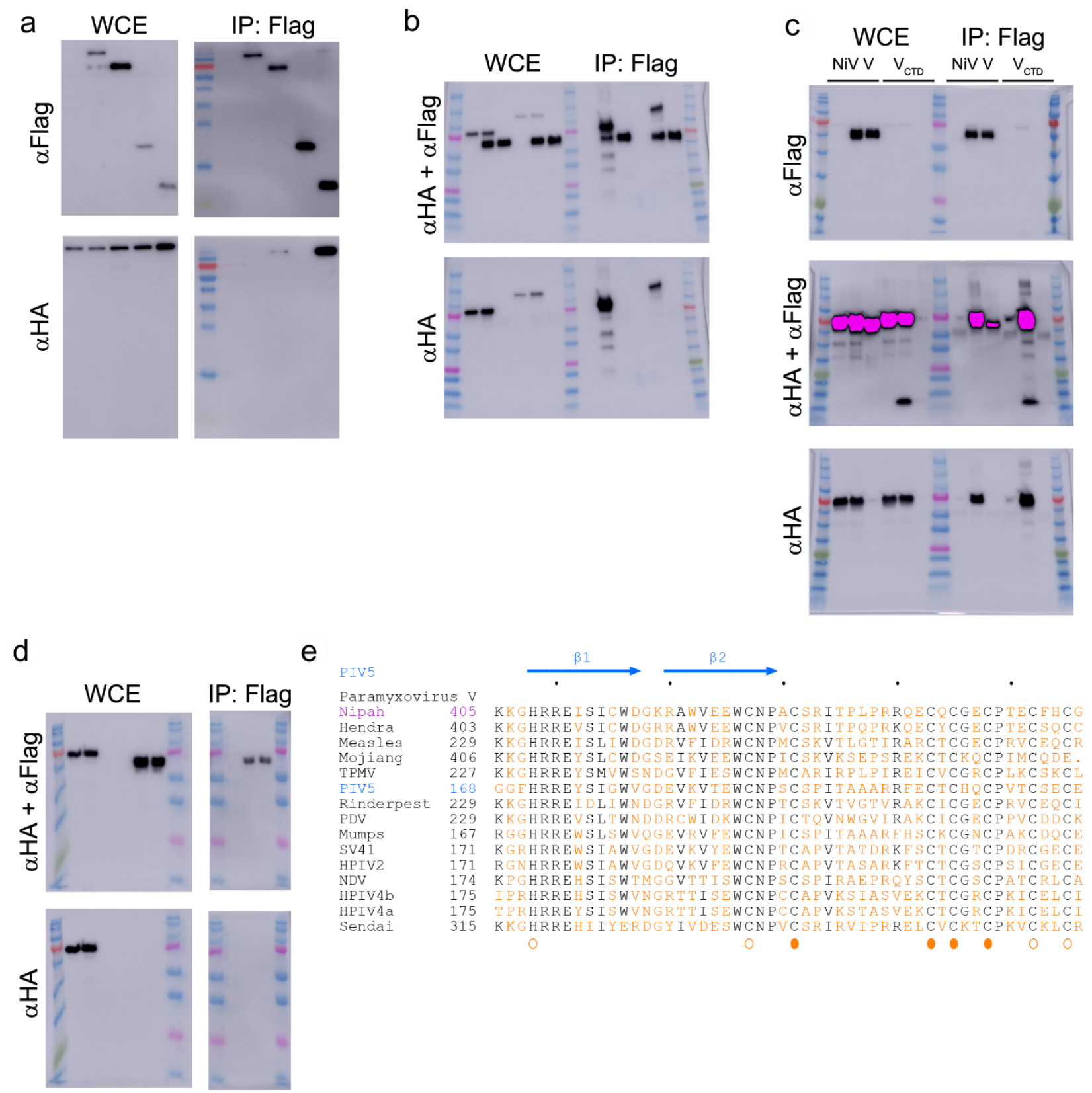

Figure S1. Corresponding western blot images used in this study, related to Figure 1. a, NiV P gene encoded protein co-IP with full-length human MDA5. b, Full-length NiV V co-IP with MDA5 domain truncations. c, Full-length NiV V and NiV V CTD Co-IP with MDA5sF2 domain. d, Individually expressed full-length NiV V co-IP with MDA5sF2 domain. e. Sequence alignment of VCTD from different paramyxoviruses, including Nipah, Hendra, Measles, Mojiang, TPMV, PIV5, Rinderpest, PDV, Mumps, SV41, HPIV2, NDV, HPIV4b, HPIV4a, and Sendai virus. Secondary structural information derived from crystal structure of MDA5sF2 in complex with PIV5 V CTD (PDB 4I1S) has been shown as reference above the sequence alignment panel. Starting residue numbers in the sequence alignment for each virus are indicated in the beginning of each sequence row. Circles below the sequence alignment panel indicated the conserved residues corresponding to $\mathrm{ZnF}$ formation. Blank circle, ZnF1. Filled circle, ZnF2. 


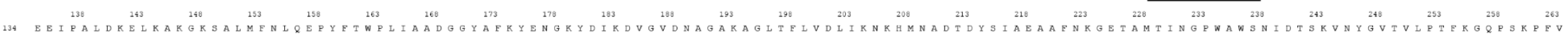

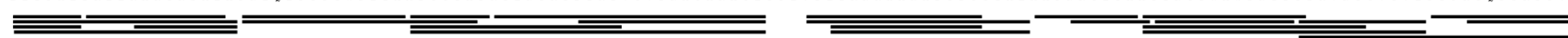

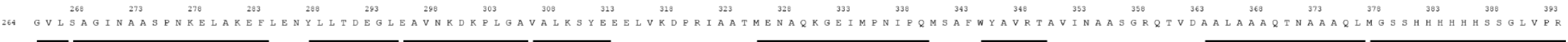

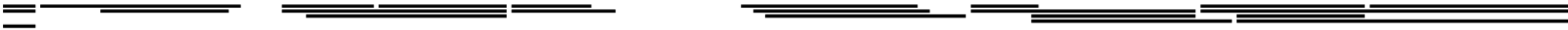

Coverage map of all identified peptides from tagged-NiV $\mathrm{V}_{\text {СтD }}$

b

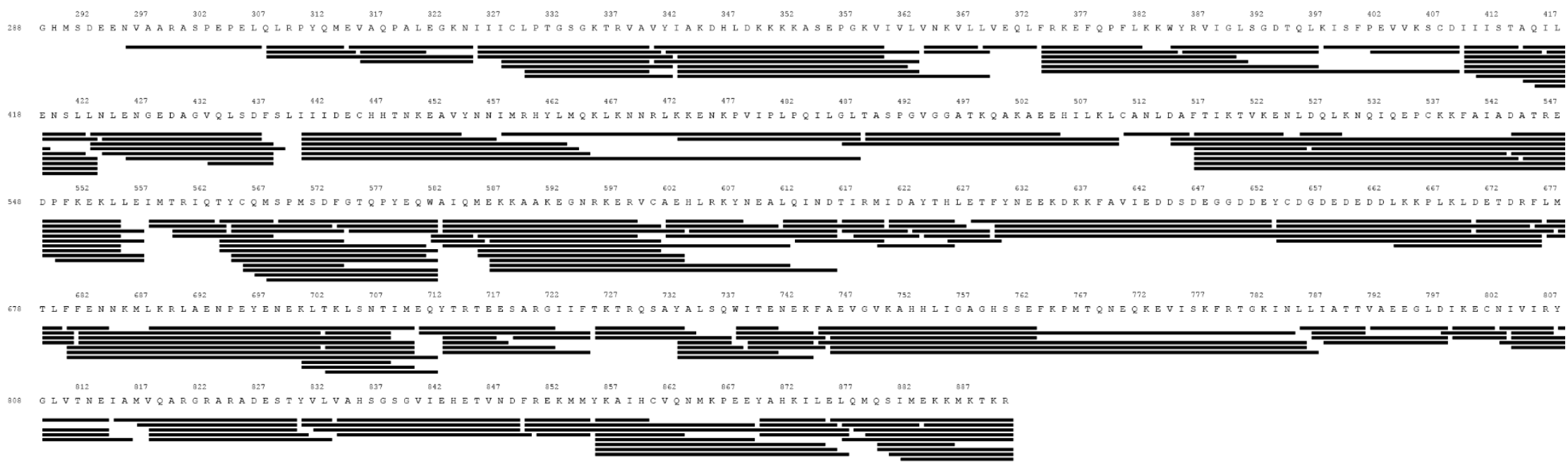

Coverage map of all identified peptides from $\mathrm{MDA}_{\mathrm{SF} 2}$

Figure S2. Coverage maps for MDA5 ${ }_{\mathrm{SF} 2}$ and tagged-NiV $\mathbf{V}_{\mathrm{CTD}}$, related to Figures 3 and 4 . a, Peptide maps showing all

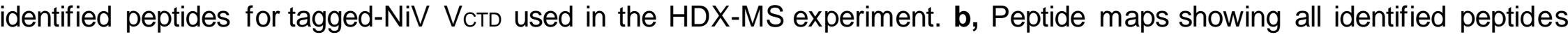
for MDA5sF2 used in the HDX-MS experiment. 


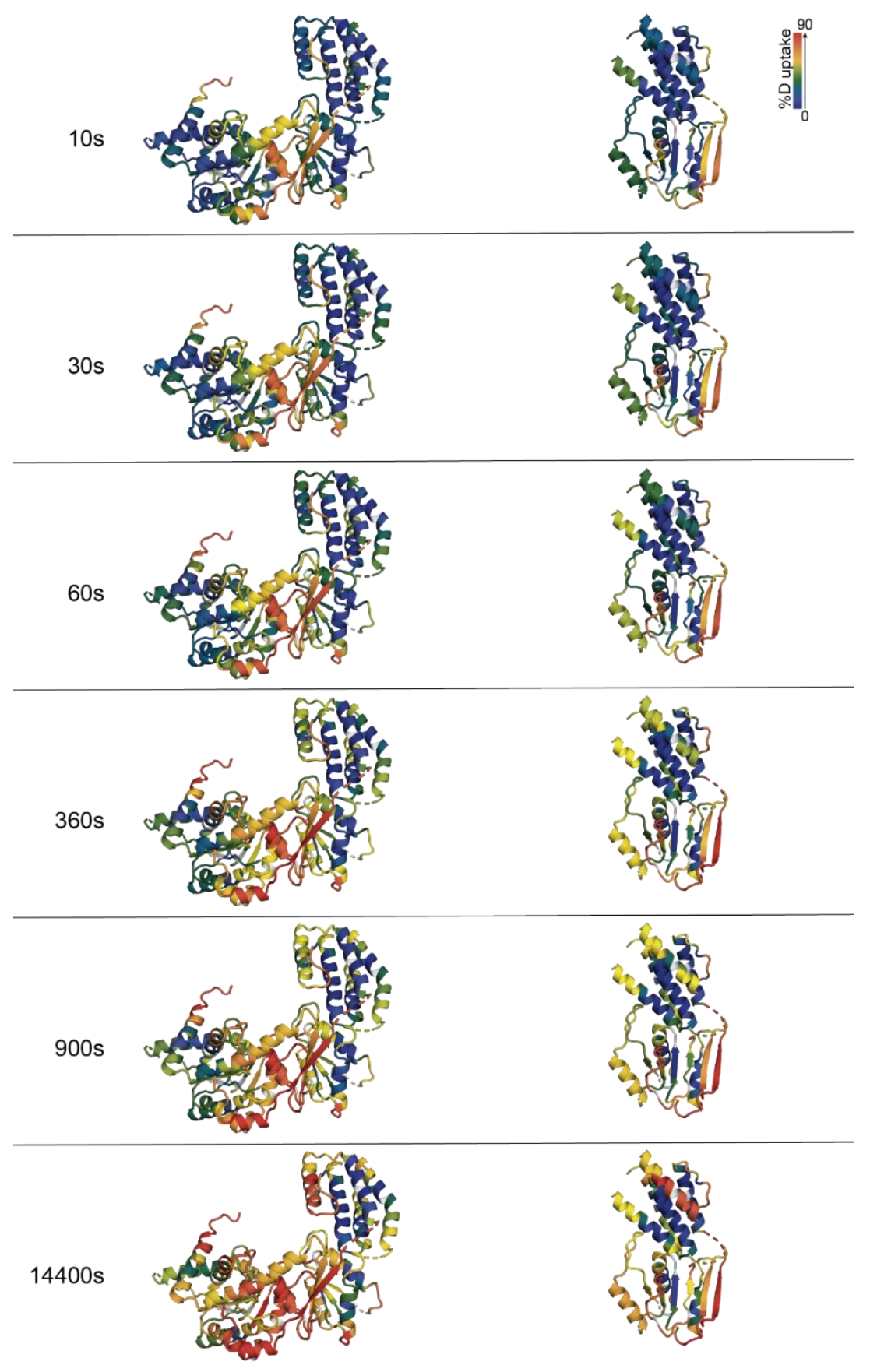

Figure S3. Unbound HDX-MS results mapped onto the structure of $\mathrm{MDA}_{\mathrm{SF} 2}$, related to Figure 4b. The HDX-MS results for all measured time points $(10 \mathrm{~s}, 30 \mathrm{~s}, 60 \mathrm{~s}, 360 \mathrm{~s}$, $900 \mathrm{~s}, 14400 \mathrm{~s}$, top to bottom, respectively) of free MDA5sF2 mapped onto the full MDA5sF2 structure ( $\triangle \mathrm{CTD}$, PDB 4GL2, left) and MDA5sF2 (displaying residues 534-809 and $827-836$ ) rotated $90^{\circ}$ (right). The extent of HDX is mapped onto the structure using the provided color bar. 

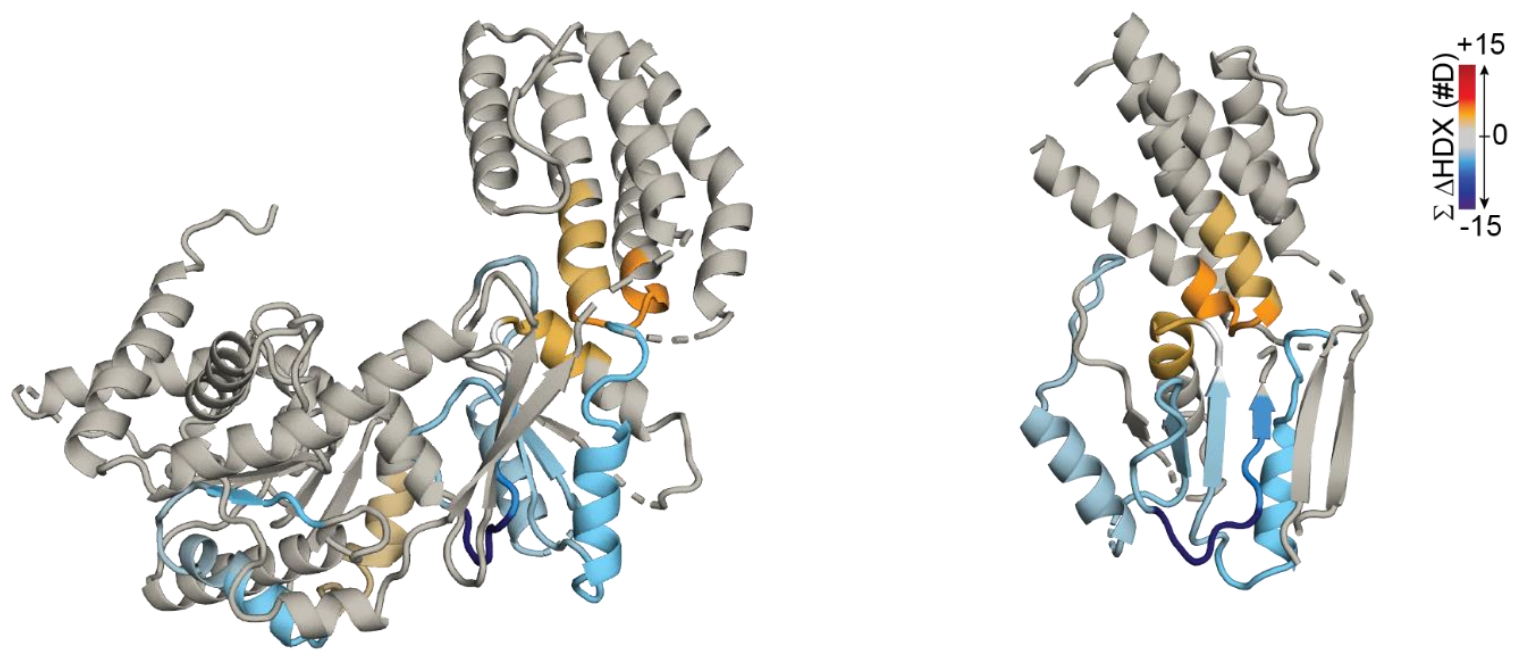

Figure S4. Differential HDX-MS results of $\mathrm{MDA}_{\mathrm{SF} 2}$ bound to NiV $\mathrm{V}_{\mathrm{CTD}}$ mapped onto the full structure, related to Figure 4c. The peptide-level differential HDX-MS results for MDA5sF2 upon binding NiV V CTD mapped onto the full MDA5sF2 structure $(\triangle C T D$, PDB 4GL2, left) and MDA5SF2 (displaying residues 534-809 and 827-836) rotated $90^{\circ}$ (right). The differential HDX extent is mapped onto the structure using the provided color bar. 